\title{
On Boundary Factors and Traces of Subgroups of Finite Groups
}

\author{
W. Guo · Alexander N. Skiba · X. Tang
}

Received: 12 September 2014 / Accepted: 4 February 2015 / Published online: 8 March 2015

(C) School of Mathematical Sciences, University of Science and Technology of China and Springer-Verlag Berlin Heidelberg 2015

\begin{abstract}
A subgroup $E$ of a finite group $G$ is called hypercyclically embedded in $G$ if every chief factor of $G$ below $E$ is cyclic. Let $A$ be a subgroup of a group $G$. Then we call any chief factor $H / A_{G}$ of $G$ a $G$-boundary factor of $A$. For any $G$-boundary factor $H / A_{G}$ of $A$, we call the subgroup $(A \cap H) / A_{G}$ of $G / A_{G}$ a $G$-trace of $A$. On the basis of these notions, we give some new characterizations of hypercyclically embedded subgroups.
\end{abstract}

Keywords Finite group · Hypercyclically embedded subgroup · $G$-boundary factor $\cdot G$-trace of subgroup $\cdot$ Meet-irreducible subgroup

Mathematics Subject Classification $\quad 20 \mathrm{D} 10 \cdot 20 \mathrm{D} 15 \cdot 20 \mathrm{D} 30$

\section{Introduction}

Throughout this paper, all groups are finite and $G$ always denotes a finite group, $p$ is supposed to be a prime.

It is obvious that a maximal subgroup $M$ of $G$ cannot be written as a proper intersection of subgroups of $G$. Proper subgroups of $G$ with this property are called primitive

\footnotetext{
W. Guo $(\bowtie) \cdot X$. Tang

School of Mathematical Sciences, University of Science and Technology of China, Hefei 230026, People's Republic of China

e-mail:wbguo@ustc.edu.cn

X. Tang

e-mail: tangxz@mail.ustc.edu.cn
}

A. N. Skiba

Department of Mathematics, Francisk Skorina Gomel State University, 246019 Gomel, Belarus e-mail: alexander.skiba49@gmail.com 
[1] or meet-irreducible [2] since, in fact, they are just the meet-irreducible elements in the sense of Birkhoff [3, p. 93] of the lattice $L(G)$ of all subgroups of G.

Recall that a normal subgroup of $G$ is called hypercyclically embedded [4, p. 217] in $G$ if either $E=1$ or $E \neq 1$ and every chief factor of $G$ below $E$ is cyclic. With study and applications of hypercyclically embedded subgroup are connected a large number of researches. Some results related to such subgroups are discussed in the books $[2,4,5]$. Among recent papers in this line of researches, see for example [6-13].

Our goal here is to give new characterizations of hypercyclically embedded subgroups on the basis of the following new notion.

Definition 1.1 Let $A$ be a proper subgroup of $G$. We call any chief factor $H / A_{G}$ of $G / H_{G}$ a $G$-boundary factor or simply boundary factor of $A$. For any $G$-boundary factor $H / A_{G}$ of $A$, we call the subgroup $(A \cap H) / A_{G}$ of $G / A_{G}$ a $G$-trace of $A$ or simply a trace of $A$.

Example 1.2 A subgroup $A$ of $G$ is said to be a $C A P$-subgroup [14, p. 37] if $A$ either covers or avoids each chief factor of $G$; a partial $C A P$-subgroup $[15,16]$ or semi $C A P$-subgroup [17] if $H$ either covers or avoids each factor of some chief series of $G$. It is clear that if $A$ is a $C A P$-subgroup of $G$, then every $G$-trace of $A$ is identity. If $A$ is a proper partial $C A P$-subgroup, then some $G$-trace of $A$ is identity. Indeed, let $1=G_{0}<G_{1}<\cdots<G_{t}=G$ be a chief series of $G$ such that $H$ either covers or avoids each factor of this series. Then there is an index $i$ such that $G_{i} \leq A$ and $G_{i+1} \not \leq A$. Hence $A$ does not cover the factor $G_{i+1} / G_{i}$, so $A$ avoids it, that is, $A \cap G_{i+1} \leq G_{i}$. Thus the trace $\left(A \cap G_{i+1}\right) / G_{i}$ is identity.

Example 1.3 Let $M$ be a maximal subgroup of $G$ and $H / M_{G}$ and $H_{1} / M_{G}$ be chief factors of $G$. Then $\left|H / M_{G}\right|=\left|H_{1} / M_{G}\right|$ by Baer's theorem [14, Ch.A, 15.2(3)], and the number $\left|H / M_{G}\right|$ is called the normal index of $M$ (Deskins). In general, the chief trace $(H \cap M) / M_{G}$ may be non-trivial (for example, if $G$ is a simple non-abelian group). But if $G$ is soluble, then every chief factor of $G$ is abelian and so any trace of $M$ is trivial by [14, Ch.A,15.2(1)(2)]. Hence in this case, the normal index of $M$ coincides with its index $|G: M|$ (Deskins).

Example 1.4 Let $G$ be $p$-soluble (respectively, $p$-supersoluble). Let $A$ be a proper subgroup of $G$ and $H / A_{G}$ be any $G$-boundary factor of $A$ such that $p$ divides $\left|H / A_{G}\right|$. Then $H / A_{G}$ is an abelian $p$-group (respectively, $H / A_{G}$ is a group of order $p$ ). Hence the trace $(H \cap A) / A_{G}$ is subnormal in $G / A_{G}$, and it is trivial in the case where $G$ is $p$-supersoluble. Therefore, if $G$ is soluble, then every trace of any proper subgroup of $G$ is subnormal; if $G$ is supersoluble, then every trace of any proper subgroup of $G$ is trivial.

Example 1.5 Every subgroup $A$ of order 2 of the alternative group $A_{4}$ of degree 4 is meet-irreducible and $A$ has a unique boundary factor which coincides with the Sylow 2-subgroup of $A_{4}$.

Recall that a subgroup $A$ of $G$ is said to permute with a subgroup $B$ if $A B=B A$.

Now we can state our first result. 
Theorem A A non-identity normal subgroup $E$ of $G$ is hypercyclically embedded in $G$ if and only if for every meet-irreducible subgroup $X$ of $E$ and some $G$-boundary factor $H / X_{G}$ of $X$, where $H \leq E$, the trace $(H \cap X) / X_{G}$ permutes with some Sylow $q$-subgroup of $G / X_{G}$ for every prime $q$ dividing $\left|G / X_{G}\right|$.

Let $\mathfrak{F}$ be a class of groups. If $1 \in \mathfrak{F}$, then we write $G^{\mathfrak{F}}$ to denote the intersection of all normal subgroups $N$ of $G$ with $G / N \in \mathfrak{F}$. A non-empty class $\mathfrak{F}$ of groups is said to be a formation provided, for every group $G$, every homomorphic image of $G / G^{\mathfrak{F}}$ belongs to $\mathfrak{F}$. A formation $\mathfrak{F}$ is said to be solubly saturated if $G \in \mathfrak{F}$ whenever $G^{\mathfrak{F}} \leq \Phi(S)$ for some normal soluble subgroup $S$ of $G$.

It is not difficult to show that if $G / E \in \mathfrak{F}$, where $\mathfrak{F}$ is a solubly saturated formation, and $E$ is hypercyclically embedded in $G$, then $G \in \mathfrak{F}$ (see [13, Lemma 2.16]). Hence, the following result follows directly from Theorem A.

Corollary 1.6 Let $\mathfrak{F}$ be a solubly saturated formation containing all supersoluble groups and $E$ a non-identity normal subgroup of $G$ with $G / E \in \mathfrak{F}$. Suppose that for every meet-irreducible subgroup $X$ of $E$ and some $G$-boundary factor $H / X_{G}$ of $X$, where $H \leq E$, the trace $(H \cap X) / X_{G}$ permutes with some Sylow $q$-subgroup of $G / X_{G}$ for every prime $q$ dividing $\left|G / X_{G}\right|$. Then $G \in \mathfrak{F}$.

Corollary $1.7 G$ is supersoluble if and only if for every meet-irreducible subgroup $X$ of $G$ and some $G$-boundary factor $H / X_{G}$ of $X$, the trace $(H \cap X) / X_{G}$ permutes with some Sylow $q$-subgroup of $G / X_{G}$ for every prime $q$ dividing $\left|G / X_{G}\right|$.

It is clear that if $|G: A|=p^{n}$, then for every prime $q \in \pi(G)$ there is a Sylow $q$-subgroup $Q$ of $G$ such that $A$ permutes with $Q$. Thus from Corollary 1.7 we get

Corollary 1.8 (See Johnson [1]). If the index $|G: X|$ is a prime power for every meet-irreducible subgroup $X$ of $G$, then $G$ is supersoluble.

The following example shows that in general the inverse of Corollary 1.8 is not true.

Example 1.9 Let $p$ and $q$ be primes such that $q$ divides $p-1$. Let $G=(\langle a\rangle \rtimes\langle b\rangle) \times\langle c\rangle$, where $|a|=|c|=p,|b|=q$ and $A=\langle a\rangle \rtimes\langle b\rangle$ is a non-abelian group of order $p q$. Then $G$ is a supersoluble group, so any $G$-trace of the subgroup $E=\langle a c\rangle$ is trivial. Moreover, $|G: E|=p q$ and $E$ does not permute with any Sylow $q$-subgroup of $G$. Hence $E$ is a meet-irreducible subgroup of $G$.

The product of all normal quasinilpotent subgroups of $G$ is denoted by $F^{*}(G)$ and it is called the generalized Fitting subgroup of $G$.

Theorem B A non-identity normal subgroup $E$ of $G$ is hypercyclically embedded in $G$ if and only iffor every two meet-irreducible subgroups $X$ and $Y$ of $E$ with $X_{G}=Y_{G}$, there are $G$-boundary factors $H / X_{G}$ and $H_{1} / X_{G}$ of $X$ and $Y$, respectively, where $H \leq E$ and $H_{1} \leq E$, such that the traces $(X \cap H) / X_{G}$ and $\left(Y \cap H_{1}\right) / X_{G}$ are conjugated by an element of the generalized Fitting subgroup $F^{*}\left(G / X_{G}\right)$.

Corollary 1.10 Let $\mathfrak{F}$ be a solubly saturated formation containing all supersoluble groups and $E$ a non-identity normal subgroup of $G$ with $G / E \in \mathfrak{F}$. Suppose that 
for every two meet-irreducible subgroups $X$ and $Y$ of $E$ with $X_{G}=Y_{G}$, there are $G$-boundary factors $H / X_{G}$ and $H_{1} / X_{G}$ of $X$ and $Y$, respectively, where $H \leq E$ and $H_{1} \leq E$, such that the traces $(X \cap H) / X_{G}$ and $\left(Y \cap H_{1}\right) / X_{G}$ are conjugated by an element of the generalized Fitting subgroup $F^{*}\left(G / X_{G}\right)$. Then $G \in \mathfrak{F}$.

It is well known [18, II, 3.2] that in a soluble group $G$ any two maximal subgroups $M$ and $L$ with $M_{G}=L_{G}$ are conjugated. It is not difficult to show that the inverse statement is also true: If any two maximal subgroups $M$ and $L$ with $M_{G}=L_{G}$ are conjugated, then $G$ is soluble (see the proof of Theorem $\mathrm{B}^{*}$ ). These observations are the motivation for our next result.

Corollary 1.11 $G$ is supersoluble if and only if for every two meet-irreducible subgroups $X$ and $Y$ of $G$ with $X_{G}=Y_{G}$, there are $G$-boundary factors $H / X_{G}$ and $H_{1} / X_{G}$ of $X$ and $Y$, respectively, such that the traces $(X \cap H) / X_{G}$ and $\left(Y \cap H_{1}\right) / X_{G}$ are conjugated by an element of the generalized Fitting subgroup $F^{*}\left(G / X_{G}\right)$.

It is clear that if $A$ is a meet-irreducible subgroup of $G$, then there is a unique subgroup $A \leq A_{0}$ of $\mathrm{G}$ such that $A$ is a maximal subgroup of $A_{0}$. We say that $A_{0}$ is the covering subgroup for $A,\left|A_{0}: A\right|$ is the small index of $A$ and denote it by $|G: A|_{0}$.

Theorem C A non-identitynormal subgroup $E$ of $G$ is hypercyclically embedded in $G$ if and only iffor every meet-irreducible subgroup $X$ of $E$ and some of its $G$-boundary factor $H / X_{G}$, where $H \leq E$, we have $|E: X|_{0}=\left|H / X_{G}\right|$.

Corollary 1.12 Let $\mathfrak{F}$ be a solubly saturated formation containing all supersoluble groups and $E$ a non-identity normal subgroup of $G$ with $G / E \in \mathfrak{F}$. Suppose that for every meet-irreducible subgroup $X$ of $E$ and some of its $G$-boundary factor $H / X_{G}$ of $X$, where $H \leq E$, we have $|E: X|_{0}=\left|H / X_{G}\right|$. Then $G \in \mathfrak{F}$.

The following result is well known (see Deskins [19]): A group $G$ is soluble if and only if the normal index $|G: M|_{n}$ of any maximal subgroup $M$ of $G$ coincides with the index $|G: M|$. Our next result is an analogue of this result for supersoluble groups.

Corollary $1.13 G$ is supersoluble if and only if for every meet-irreducible subgroup $X$ of $G$ and some of its $G$-boundary factor $H / X_{G}$, we have $|E: X|_{0}=\left|H / X_{G}\right|$.

\section{Proofs of Theorems A, B and C}

The following results are useful in our proof.

Lemma 2.1 (See [2, Ch. 4, 5.1])). Let $R \leq A \leq G$, where $R$ is normal in $G$.

(1) If $A \leq B \leq G$, where $A$ is a meet-irreducible subgroup of $B$, then there is a meet-irreducible subgroup $X$ of $G$ such that $A=B \cap X$.

(2) $A$ is a meet-irreducible subgroup of $G$ if and only if $A / R$ is a meet-irreducible subgroup of $G / R$.

Lemma 2.2 (See [20]). Let $H, K$ and $N$ be pairwise permutable subgroups of $G$ and $H$ is a Hall subgroup of $G$. Then $N \cap H K=(N \cap H)(N \cap K)$. 
Recall that the largest normal subgroup $E$ of $G$ such that every chief factor of $G$ below $E$ is cyclic is called the $\mathfrak{U}$-hypercentre of $G$ and is denoted by $Z_{\mathfrak{U}}(G)$ (see [14, p. 389]).

Theorem $\mathrm{A}$ is a corollary of the following more general result since $\cap_{p_{i} \in \pi(E)} O_{p_{i}^{\prime}}(E)$ $=1$.

Theorem $\mathbf{A}^{*}$ Let $E$ be a non-identity normal subgroup of $G$. Then $E / O_{p^{\prime}}(E) \leq$ $Z_{\mathfrak{U}}\left(G / O_{p^{\prime}}(E)\right)$ if and only if for every meet-irreducible subgroup $X$ of $E$ such that $p$ divides the order of some $G$-boundary factor $H / X_{G}$ of $X$, where $H \leq E$, the trace $(H \cap X) / X_{G}$ permutes with some Sylow $q$-subgroup of $G / X_{G}$ for all primes $q$ dividing $\left|G / X_{G}\right|$.

Proof Sufficiency. Assume that this is false and let $G$ be a counterexample with $|G|+$ $|E|$ minimal. Let $R$ be a minimal normal subgroup of $G$ contained in $E$. Then

(1) The hypothesis holds for $(G / R, E / R)$. Hence $(E / R) / O_{p^{\prime}}(E / R) \leq Z_{\mathfrak{U}}((G / R)$ $\left./ O_{p^{\prime}}(E / R)\right)$.

Let $X / R$ be any meet-irreducible subgroup of $G / R$ such that $p$ divides the order of some $(G / R)$-boundary factor $(H / R) /(X / R)_{G / R}$ of $X / R$, where $H / R \leq E / R$. Let $q$ be any prime dividing $\left|(G / R) /(X / R)_{G / R}\right|=\left|(G / R) /\left(X_{G} / R\right)\right|=$ $\left|G / X_{G}\right|$. Then, by hypothesis, there is a Sylow $q$-subgroup $Q / X_{G}$ of $G / X_{G}$ such that

$$
\begin{aligned}
Q(H \cap X) / X_{G} & =\left(Q / X_{G}\right)\left((H \cap X) / X_{G}\right) \\
& =\left((H \cap X) / X_{G}\right)\left(Q / X_{G}\right)=(H \cap X) Q / X_{G},
\end{aligned}
$$

so

$$
(Q(H \cap X) / R) /\left(X_{G} / R\right)=((H \cap X) Q / R) /\left(X_{G} / R\right) .
$$

Hence

$$
\begin{aligned}
& \left((Q / R) /(X / R)_{G / R}\right)\left(((H / R) \cap(X / R)) /(X / R)_{G / R}\right) \\
& \quad=\left(((H / R) \cap(X / R)) /(X / R)_{G / R}\right)\left((Q / R) /(X / R)_{G / R}\right)
\end{aligned}
$$

where $(Q / R) /(X / R)_{G / R}=(Q / R) /\left(X_{G} / R\right)$ is a Sylow $q$-subgroup of $(G / R) /(X / R)_{G / R}$. Therefore the hypothesis holds for $(G / R, E / R)$. The choice of $G$ implies that $(E / R) / O_{p^{\prime}}(E / R) \leq Z_{\mathfrak{U}}\left((G / R) / O_{p^{\prime}}(E / R)\right)$.

(2) $O_{p^{\prime}}(E)=1$. Hence $p$ divides $|R|$.

Assume that $O_{p^{\prime}}(E) \neq 1$. Without loss of generality, we can assume that $R \leq O_{p^{\prime}}(E)$. Then $O_{p^{\prime}}(E / R)=O_{p^{\prime}}(E) / R$. Claim (1) implies that $(E / R) / O_{p^{\prime}}(E / R) \leq Z_{\mathfrak{U}}\left((G / R) / O_{p^{\prime}}(E / R)\right)$. On the other hand, from the $G$ isomorphism $E / O_{p^{\prime}}(E) \simeq(E / R) /\left(O_{p^{\prime}}(E) / R\right)$ it follows that every chief factor of $G$ between $E$ and $O_{p^{\prime}}(E)$ is cyclic. Hence $E / O_{p^{\prime}}(E) \leq Z_{\mathfrak{U}}\left(G / O_{p^{\prime}}(E)\right)$, contrary to the choice of $G$. Therefore $O_{p^{\prime}}(E)=1$ and so $p$ divides $|R|$. 
(3) If $R$ is the only minimal normal subgroup of $G$ contained in $E$ and $X$ is a meetirreducible subgroup of $E$, then $X \cap R$ permutes with some Sylow $q$-subgroup $Q$ of $G$ for every prime $q$ dividing $|G|$.

If $R \leq X$, it is evident. Now assume that $R \not X$. Then $X_{G}=1$. Since $R$ is the unique minimal normal subgroup of $G$ contained in $E, R / 1$ is the unique $G$-boundary of $X$. Therefore, by hypothesis, there is a Sylow $q$-subgroup $Q$ of $G$ such that $(X \cap R) Q=Q(X \cap R)$.

(4) $C_{E}(R) \neq 1$. Hence $E$ is $p$-soluble by (1) and $R$ is a $p$-group.

If $G$ has two different minimal normal subgroups contained in $E$, then it follows from Claim (1). We may, therefore, assume that $R$ is the unique minimal normal subgroup of $G$ contained in $E$. Now, in view of Claims (1) and (2), it is enough to show that $R$ is abelian. Suppose that this is false. Then by the Feit-Thompson theorem, 2 divides $|R|$ and $R$ is not a 2-group. Let $R_{0}=P_{1} \cap R$ be a Sylow 2-subgroup of $R$, where $P_{1}$ is some Sylow 2-subgroup of $G$, and $V$ a maximal subgroup of $R_{0}$. Then $N_{R}(V) / V$ is 2-nilpotent by [18, IV,2.8], so $N_{R}(V)$ has a subgroup $W$ such that $\left|N_{R}(V): W\right|=2$ and $V \leq W$. Then $W$ is a meetirreducible subgroup of $N_{R}(V)$. Hence by Lemma 2.1, there is a meet-irreducible subgroup $X_{0}$ of $R$ such that $W=X_{0} \cap N_{R}(V)$. Clearly, 2 divides $\left|R: X_{0}\right|$ and 4 does not divide $\left|R: X_{0}\right|$. Again applying Lemma 2.1, we obtain that $X_{0}=X \cap R$ for some meet-irreducible subgroup $X$ of $G$.

Claim (3) implies that $G$ has a Sylow 2-subgroup $P$ such that $X_{0} P=P X_{0}$. Let $R_{2}=P \cap R$ be a Sylow 2-subgroup of $R$. Then $X_{0} P \cap R=X_{0}(P \cap R)=X_{0} R_{2}$ is a subgroup of $R$, and $X_{0}<X_{0} R_{2}$ since 2 divides $\left|R: X_{0}\right|$.

Suppose that some prime $q \neq 2$ divides $\left|R: X_{0}\right|$. Since $R$ is the unique minimal normal subgroup of $G$ contained in $E$, Claim (3) implies that there is a Sylow $q$-subgroup $Q$ of $G$ such that $X_{0} Q=Q X_{0}$. Then $X_{0} Q \cap R=X_{0}(Q \cap R)$ is a subgroup of $R$ and $X_{0}<X_{0} R_{q}$, where $R_{q}=Q \cap R$ is a Sylow $q$-subgroup of $R$. It is also clear that $X_{0} Q \cap X_{0} R_{2}=X_{0}$. Hence $X_{0}$ is not a meet-irreducible subgroup of $R$. This contradiction shows that $\left|R: X_{0}\right|=2$, so $X_{0}$ is normal in $R$. It follows that $1 \neq O^{2}(R) \neq R$. Since $O^{2}(R)$ is characteristic in $R$, it is normal in $G$, which contradicts the minimality of $R$. Hence $R$ is an abelian $p$-group.

(5) If $R$ is the only minimal normal subgroup of $G$ contained in $E$, then $|R|=p$.

In view of Claim (4), $R$ is a $p$-group. Let $V$ be a maximal subgroup of $R$ such that $V$ is normal in a Sylow $p$-subgroup $G_{p}$ of $G$. Let $q \neq p$ be any other prime dividing $|G|$. By Lemma 2.1, there is a meet-irreducible subgroup $X$ of $G$ such that $V=X \cap R$. Claim (3) implies that for some Sylow $q$-subgroup $Q$ of $G$ we have $V Q=Q V$. It is also clear that $V=V Q \cap R$ is normal in $V Q$, so $q$ does not divide $\left|G: N_{G}(V)\right|$. Hence $V$ is normal in $G$ and therefore $|R|=p$.

(6) If $R \leq \Phi(G)$, then $O_{p^{\prime}}(E / R)=1$ and so $E / R \leq Z_{\mathfrak{U}}(G / R)$.

Claim (1) implies that $(E / R) / O_{p^{\prime}}(E / R) \leq Z_{\mathfrak{U}}\left((G / R) / O_{p^{\prime}}(E / R)\right)$. Let $V / R=$ $O_{p^{\prime}}(E / R)$. Clearly, $V$ is $p$-soluble, so by the Frattini argument we have $G=$ $V N_{G}(S)=R S N_{G}(S)=N_{G}(S)$, where $S$ is a Hall $p^{\prime}$-subgroup of $V$. But this contradicts Claim (2). Hence $O_{p^{\prime}}(E / R)=1$, and so $E / R \leq Z_{\mathfrak{U}}(G / R)$.

(7) If for some minimal normal subgroup $L$ of $G$ we have $L \leq E$ and $L \neq R$, then $R$ and $L$ are cyclic p-groups. 
First note that $p$ divides $|L|$ by Claim (2). On the other hand, by Claim (1), $(E / R) / O_{p^{\prime}}(E / R) \leq Z_{\mathfrak{U}}\left((G / R) / O_{p^{\prime}}(E / R)\right)$, so from the $G$-isomorphism $L \simeq$ $L R / R$ we see that $L$ is either a $p^{\prime}$-group or a cyclic group. Hence $L$ must be a cyclic $p$-group. Similarly, $R$ is cyclic $p$-groups.

(8) $\Phi(G) \cap O_{p}(E)=1$.

Assume that $\Phi(G) \cap O_{p}(E) \neq 1$. Then $E$ has a minimal normal subgroup $R \leq \Phi(G)$. Hence $E / R \leq Z_{\mathfrak{U}}(G / R)$ by Claim (6). But by Claims (5) and (7), $R$ is cyclic. It follows that $E \leq Z_{\mathfrak{U}}(G)$. This contradiction shows that we have (8). Final contradiction for the sufficiency.

Since $\Phi\left(O_{p}(E)\right) \leq \Phi(G)$, Claim (8) implies that $\Phi\left(O_{p}(E)\right)=1$, so $O_{p}(E)$ is abelian. Therefore, again by Claim (8), for every minimal normal subgroup $N$ of $G$ contained in $O_{p}(E)$, there is a maximal subgroup $M$ of $G$ such that $O_{p}(E)=N \times$ $\left(O_{p}(E) \cap M\right)$, where $O_{p}(E) \cap M$ is normal in $G$. Hence $O_{p}(E)=N_{1} \times \cdots \times N_{t}$ for some minimal normal subgroups $N_{1}, \ldots, N_{t}$ of $G$. Let $C_{i}=C_{G}\left(N_{i}\right)$. Then $C=C_{G}\left(O_{p}(E)\right)=C_{1} \cap \cdots \cap C_{t}$. Since $E$ is $p$-soluble by Claim (4) and $O_{p^{\prime}}(E)=1$ by Claim (2), $O_{p}(E)=C \cap E$ by [18, VI,6.5]. Note that $\left|N_{i}\right|=p$ for all $i=1, \ldots t$. Indeed, if $t>1$, then it follows from Claim (7). Suppose that $t=1$. Then Claims (2), (4) and (7) imply that $N_{1}=R$ is a unique minimal normal subgroup of $G$ contained in $E$, so $\left|N_{1}\right|=p$ by Claim (5). Hence $G / C_{i}$ is cyclic for all $i=1, \ldots, t$. This implies that $G / C$ is an abelian group. Therefore from the $G$-isomorphism $C E / C \simeq E / C \cap E=E / O_{p}(E)$, we see that every chief factor of $G$ between $E$ and $O_{p}(E)$ is cyclic. Thus $E \leq Z_{\mathfrak{U}}(G)$ by the JordanHölder theorem [14, Ch.A,3.2]. The final contradiction completes the proof of the sufficiency.

Necessity. Let $X$ be any meet-irreducible subgroup of $E$ such that $p$ divides the order of some $G$-boundary factor $H / X_{G}$ of $X$, where $H \leq E$. Since $E / O_{p^{\prime}}(E) \leq$ $Z_{\mathfrak{U}}\left(G / O_{p^{\prime}}(E)\right), E$ is $p$-supersoluble, so $\left|H / X_{G}\right|=p$. Hence $(H \cap X) / X_{G}=1$. The theorem is proved.

A group $G$ is called semisimple if either $G=1$ or $G$ is the direct product of some simple non-abelian groups.

Theorem B is a corollary of the following our result.

Theorem B* Let $E$ be a non-identity normal subgroup of $G$. Then $E / O_{p^{\prime}}(E) \leq$ $Z_{\mathfrak{U}}\left(G / O_{p^{\prime}}(E)\right)$ if and only if for every two meet-irreducible subgroups $X$ and $Y$ of $E$ with $X_{G}=Y_{G}$ such that $p$ divides the order of some $G$-boundary factors $H / X_{G}$ and $H_{1} / X_{G}$ of $X$ and $Y$ respectively, where $H \leq E$ and $H_{1} \leq E$, the traces $(X \cap H) X_{G} / X_{G}$ and $\left(Y \cap H_{1}\right) X_{G} / X_{G}$ are conjugated by an element of the generalized Fitting subgroup $F^{*}\left(G / X_{G}\right)$.

Proof Sufficiency. Assume that this is false and let $G$ be a counterexample with $|G|+$ $|E|$ minimal. Let $R$ be a minimal normal subgroup of $G$ contained in $E$. We first show that the hypothesis holds for $(G / R, E / R)$.

Let $X / R$ and $Y / R$ be meet-irreducible subgroups of $E / R$ with $(X / R)_{G / R}=$ $(Y / R)_{G / R}$ such that $p$ divides the orders of some $(G / R)$-boundary factors $(H / R) /$ $(X / R)_{G / R}$ and $\left(H_{1} / R\right) /(X / R)_{G / R}$ of $X / R$ and $Y / R$ respectively, where $H / R \leq E / R$ and $H_{1} / R \leq E / R$. Then $X$ and $Y$ are meet-irreducible subgroups of $E$ with $X_{G}=Y_{G}$ 
such that $p$ divides the orders of the $G$-boundary factors $H / X_{G}$ and $H_{1} / X_{G}$ of $X$ and $Y$ respectively, where $H \leq E$ and $H_{1} \leq E$. By hypothesis, for some element $x X_{G} \in F^{*}\left(G / X_{G}\right)$ we have

$$
\left((X \cap H) / X_{G}\right)^{x X_{G}}=\left(Y \cap H_{1}\right) / X_{G} .
$$

Let $f: G / X_{G} \rightarrow(G / R) /\left(X_{G} / R\right)$ be the natural isomorphism. Then

$$
f\left(x X_{G}\right)=x R\left(X_{G} / R\right) \in F^{*}\left((G / R) /\left(X_{G} / R\right)\right)
$$

and

$$
\begin{aligned}
\left.f\left(\left((X \cap H) / X_{G}\right)\right)^{x X_{G}}\right) & =\left(((X \cap H) / R) /\left(X_{G} / R\right)\right)^{x R\left(X_{G} / R\right)} \\
& \left.=((X / R) \cap(H / R)) /(X / R)_{G / R}\right)^{x R\left(X_{G} / R\right)} \\
& =f\left(\left(Y \cap H_{1}\right) / X_{G}\right)=\left((Y / R) \cap\left(H_{1} / R\right)\right) /(X / R)_{G / R} .
\end{aligned}
$$

Therefore the hypothesis holds for $(G / R, E / R)$.

Then $O_{p^{\prime}}(E)=1$ and so $p$ divides $|R|$ (see Claim (2) in the proof of Theorem $A^{*}$ ). Moreover, if for some minimal normal subgroup $L$ of $G$ we have $L \leq E$ and $L \neq R$, then $R$ and $L$ are cyclic $p$-groups (see Claim (6) in the proof of Theorem $A^{*}$ ).

Now assume that $R$ is the only minimal normal subgroup of $G$ contained in $E$. We now show that $|R|=p$. First we claim that $R$ is a $p$-group. Assume that this is false. Then $R$ is a non-abelian group, so the Frattini argument implies that, for any prime $q$ dividing $|R|$, there is a maximal subgroup $L$ of $E$ such that $R \not L L$ and a Sylow $q$-subgroup $R_{q}$ of $R$ is contained in $R \cap L$. On the other hand, if $M$ is a maximal subgroup of $E$ such that $R \not M M$, then $R / 1=R / M_{G}$ is the only $G$-boundary factor of $M$. Hence for any two maximal subgroups $M$ and $L$ not containing $R$, we have $|M \cap R|=|L \cap R|$ by hypothesis. It follows that $R \cap M=R$, a contradiction. Thus $R$ is a $p$-group.

Next we show that $|R|=p$. Indeed, assume that $|R|>p$. Then it has two different maximal subgroups $V$ and $W$. Hence $V$ and $W$ are meet-irreducible subgroups of $R$. By Lemma 2.1, there are meet-irreducible subgroups $X$ and $Y$ of $E$ such that $V=X \cap R$ and $W=Y \cap R$. Since $R$ is the only minimal normal subgroup of $G$ contained in $E, R / 1=R / M_{G}$ is the only $G$-boundary factor of $V$ and $W$ satisfying $R \leq E$. Therefore by hypothesis there is an element $x \in F^{*}(G)$ such that $W=V^{x}$. If $F^{*}(G) \cap E=1$, then $F^{*}(G) \leq C_{G}(E)$ and so $W=V^{x}=V$, a contradiction. Hence $F^{*}(G) \cap E \neq 1$. But then $R \leq F^{*}(G) \cap E$. By [21, X, 13.6], $F^{*}(G) / Z_{\infty}\left(F^{*}(G)\right)$ is a semisimple group. Since $R$ is a $p$-group, it follows that $R \leq Z_{\infty}\left(F^{*}(G)\right)$. Thus $F^{*}(G) / C_{F^{*}(G)}(R)$ is a $p$-group by $[22, C h .5,3.2]$. On the other hand, $Z_{\infty}\left(F^{*}(G)\right)=$ $F(G)$ by [21, X, 13.7]. Hence $Z_{\infty}\left(F^{*}(G)\right) \leq C_{G}(R)$ by [2, AppendixC, 3.2]. But then we have $C_{F^{*}(G)}(R)=F^{*}(G)$ and so again we get that $W=V$. This contradiction shows that $|R|=p$.

The above shows that every minimal normal subgroup of $G$ contained in $E$ is a cyclic $p$-group. Now, the final part of the proof of the sufficiency can be proved similarly as the final part of the proof of the sufficiency in Theorem $A^{*}$. 
Necessity. Let $X$ and $Y$ be any two meet-irreducible subgroups with $X_{G}=Y_{G}$ such that $p$ divides the order of some $G$-boundary factors $H / X_{G}$ and $H_{1} / X_{G}$ of $X$ and $Y$, respectively, where $H \leq E$ and $H_{1} \leq E$. Then, since $E / O_{p^{\prime}}(E) \leq$ $Z_{\mathfrak{U}}\left(G / O_{p^{\prime}}(E)\right),\left|H / X_{G}\right|=p=\left|H_{1} / X_{G}\right|$ and so the traces $(X \cap H) / X_{G}$ and $\left(Y \cap H_{1}\right) / X_{G}$ are the identities.

The theorem is proved.

Lemma 2.3 Let $R$ be a normal subgroup and A a meet-irreducible subgroup of $G$.

(1) If $R \leq A$, then $|G: A|_{0}=|G / R: A / R|_{0}$.

(2) If $R \not \leq A$, then $A_{0}=A\left(A_{0} \cap R\right)$ and $|G: A|_{0}=\left|\left(A_{0} \cap R\right):(A \cap R)\right|$.

Proof Both assertions are evident. Indeed, if, for example, $R \not A A$, then $A<A R$ and so $A_{0} \leq A R$. Hence $A_{0}=A\left(A_{0} \cap R\right)$. It follows that $|G: A|_{0}=\left|A_{0}: A\right|=$ $\left|\left(A_{0} \cap R\right):\left(A \cap A_{0} \cap R\right)\right|=\left|\left(A_{0} \cap R\right):(A \cap R)\right|$.

Theorem $\mathrm{C}$ is a corollary of the following result.

Theorem $C^{*}$ Let $E$ be a non-identity normal subgroup of $G$. Then $E / O_{p^{\prime}}(E) \leq$ $Z_{\mathfrak{U}}\left(G / O_{p^{\prime}}(E)\right.$ if and only if for every meet-irreducible subgroup $X$ of $E$ such that $p$ divides the order of some $G$-boundary factor $H / X_{G}$ of $X$, where $H \leq E$, we have $|E: X|_{0}=\left|H / X_{G}\right|$.

Proof Sufficiency. Assume that this is false and let $G$ be a counterexample with $|G|+$ $|E|$ minimal. Let $R$ be a minimal normal subgroup of $G$ contained in $E$.

We first show that the hypothesis holds for $(G / R, E / R)$. Indeed, let $X / R$ be a meetirreducible subgroup of $E / R$ such that $p$ divides the order of a $(G / R)$-boundary factor $(H / R) /(X / R)_{G / R}$ of $X / R$ satisfying $H / R \leq E / R$. Then $X$ is a meet-irreducible subgroup of $E$ and $H / X_{G}$ is a $G$-boundary factor of $X$ such that $H \leq E$ and $p$ divides $\left|H / X_{G}\right|$. Hence $|E: X|_{0}=\left|H / X_{G}\right|$ by hypothesis, so

$$
|E / R: X / R|_{0}=|E: X|_{0}=\left|(H / R) /\left(X_{G} / R\right)\right|=\left|(H / R) /(X / R)_{G / R}\right| .
$$

This shows that the hypothesis holds for $(G / R, E / R)$. It follows that $O_{p^{\prime}}(E)=1$ and so $p$ divides $|R|$ (see Claim (2) in the proof of Theorem $A^{*}$ ).

If for some minimal normal subgroup $L$ of $G$ we have $L \leq E$ and $L \neq R$, then $R$ and $L$ are cyclic $p$-groups (see Claim (7) in the proof of Theorem $A^{*}$ ).

Now assume that $R$ is the only minimal normal subgroup of $G$ contained in $E$. If $R$ is a non-abelian group, then for some Sylow $p$-subgroup $R_{p}$ of $R$ we have $N_{E}\left(R_{p}\right) \neq E$. Let $M$ be a maximal subgroup of $E$ such that $N_{E}\left(R_{p}\right) \leq M$. The Frattini argument implies that $R \not M M$. Then, in view of the $G$-isomorphism $R M_{G} / M_{G} \simeq R$, we have that $R M_{G} / M_{G}$ is a $G$-boundary factor of $M$ such that $p$ divides $\left|R M_{G} / M_{G}\right|$ and $R M_{G} \leq E$. The hypothesis implies that $\left|R M_{G} / M_{G}\right|=|E: M|_{0}=|E: M|$. Hence $p$ divides $|E: M|$. But for a Sylow $p$-subgroup $E_{p}$ of $E$ containing $R_{p}$ we have $E_{p} \cap R=R_{p}$, so $E_{p} \leq N_{E}\left(R_{p}\right) \leq M$. Hence $p$ does not divide $|E: M|$. This contradiction show that $R$ is a $p$-group.

Now we show that $|R|=p$. Assume that this is false. Let $V$ be a maximal subgroup of $R$. Then $V \neq 1$ and, by Lemma 2.1, for some meet-irreducible subgroup $X$ of $E$ we 
have $V=X \cap R$. It is clear that $R \not X X$, so $X_{G}=1$ since $R$ is the only minimal normal subgroup of $G$ contained in $E$. Hence $R / 1=R / M_{G}$ is the only $G$-boundary factor of $X$ satisfying $R \leq E$. Thus $|E: X|_{0}=|R|$ by hypothesis. But $|E: X|_{0}=|R / V|=p$, so $V=1$. This contradiction shows that $|R|=p$.

The above shows that every minimal normal subgroup of $G$ contained in $E$ is a cyclic $p$-group. Now, the final part of the proof of the sufficiency can be proved similarly as the final proof of the sufficiency in Theorem $A^{*}$.

Necessity. Let $X$ be any meet-irreducible subgroup of $E$ such that $p$ divides the order of some $G$-boundary factor $H / X_{G}$ of $X$ where $H \leq E$. Since $E / O_{p^{\prime}}(E) \leq$ $Z_{\mathfrak{U}}\left(G / O_{p^{\prime}}(E)\right), E$ is $p$-supersoluble. This implies that $\left|H / X_{G}\right|=p$. Hence by Lemma 2.3(2), $1 \neq|E: X|_{0}=\left|H \cap X_{0}: X \cap H\right|=\left|H \cap X_{0}: X_{G}\right|$. Consequently $|E: X|_{0}=\left|H / X_{G}\right|$. The theorem is thus proved.

\section{Some Other Applications}

In this section, we discuss some other applications of the notions of boundary factor and trace of a subgroup.

1. Recall that a series $M<T<G$, where $T$ is a maximal subgroup of $G$ and $M$ is a maximal subgroup of $T$, is said to be a maximal chain of $G$ of length 2 .

A large number of results are based on a stronger condition for subgroups "cover or avoid chief factors". For example, it is known that if either every maximal subgroup of $G$ is a (partial) $C A P$-subgroup of $G$ or every second maximal subgroup of $G$ is a (partial) $C A P$-subgroup of $G$ (see $[17,23]$ ), then $G$ is soluble. Note that the subgroup $A$ in Example 1.5 is 2-maximal and it is not a partial $C A P$ subgroup of $A_{4}$.

Nevertheless, we can prove the following theorem which contains the abovementioned results in [17,23].

Theorem 3.1 $G$ is soluble if and only if every maximal chain of $G$ of length 2 contains a proper subgroup $M$ of $G$ such that some $G$-trace of $M$ is subnormal.

In order to prove Theorem 3.1, the following well-known result (see [24, (6.6.3)]) is used.

Lemma 3.2 Let $G=R \rtimes M$. If $M$ is a soluble maximal subgroup of $G$, then $R$ is abelian.

Proof of Theorem 3.1. In view of Example 1.4, it is enough to prove that if every maximal chain of $G$ of length 2 contains a proper subgroup $M$ of $G$ such that some $G$-trace of $M$ is subnormal, then $G$ is soluble. Assume that this is false and let $G$ be a counterexample of minimal order. Let $R$ be a minimal normal subgroup of $G, q$ the largest prime dividing $|R|$ and $R_{q}$ a Sylow $q$-subgroup of $R$. Then:

(1) $G / R$ is soluble. Hence $R$ is the unique minimal normal subgroup of $G, R \nsubseteq$ $\Phi(G), C_{G}(R)=1$ and so $q>3$.

Let $M / R<T / R<G / R$ be any maximal chain of $G / R$ of length 2 . Then $M<T<G$ is a maximal chain of $G$ of length 2, so for one of the subgroups $M$ 
or $T$ (we denote it by $L$ ) some $G$-trace $\left(H_{1} \cap L\right) / L_{G}$ of $L$ is subnormal in $G / L_{G}$ by hypothesis. But then the $(G / R)$-trace

$$
\left(\left(H_{1} / R\right) \cap(L / R)\right) /(L / R)_{G / R}=\left(\left(H_{1} \cap L\right) / R\right) /\left(L_{G} / R\right)
$$

of $L / R$ is subnormal in $(G / R) /(L / R)_{G / R}$. This shows that the hypothesis holds for $G / R$. The choice of $G$ implies that $G / R$ is soluble, and it follows that $R$ is the unique minimal normal subgroup of $G, R \nsubseteq \Phi(G), C_{G}(R)=1$. It is clear that $2 \in \pi(R)$ and $q>3$ by the well-known Feit-Thompson theorem and Burnside $p^{a} q^{b}$-theorem.

(2) For some maximal subgroup $M$ of $G$ and some Sylow $q$-subgroup $G_{q}$ of $G$, we have $R_{q} \leq G_{q} \leq N_{G}\left(R_{q}\right) \leq M$ and $M_{G}=1$. (This follows from Claim (1) and Frattini argument).

(3) $R \neq G$, so $D=M \cap R \neq M$.

Suppose that $R=G$ is a simple non-abelian group. Then $G / 1$ is the only $G$ boundary factor of any proper subgroup of $G$. By Claim (1), $G$ is not 2-nilpotent and so $G$ has a 2-closed Schmidt subgroup $H=H_{2} \rtimes H_{t}$ by [18, IV, 5.4]. It is clear that $\left|H_{2}\right| \neq 2$ and $H \neq G$. Hence $G$ has a maximal chain $T<L<G$, where $T \neq 1$. Since $p$ divides $|G|$, at least one of the subgroups $T$ or $L$ is a proper non-identity subnormal subgroup of $G$ by hypothesis, so $G$ is not a simple nonabelian group. This contradiction shows that $R \neq G$. Since $M_{G}=1, M R=G$ and so $D=M \cap R \neq M$.

(4) $D$ is a normal non-nilpotent subgroup of $M$, so $D \not \leq \Phi(M)$.

Clearly, $R_{q}$ is a Sylow subgroup of $D$. Suppose that $D$ is nilpotent. Then $R_{q}$ is a characteristic subgroup of $D$, so $M \leq N_{G}\left(R_{q}\right)$ since $D$ is normal in $M$. Hence, in view of Claim (1), we have $M=N_{G}\left(R_{q}\right)$ and so $N_{R}\left(R_{q}\right)=D$ is nilpotent. Then $N_{R}\left(R_{q}\right) / C_{R}\left(R_{q}\right)$ is a $q$-group and hence $O^{q}(R) \neq R$ by [21, $\left.\mathrm{X}, 8.13\right]$ since $q>3$ by Claim(1). But in view of Claim (1) again, every composition factor of $R$ is non-abelian, a contradiction. Thus we have (4).

(5) $M$ has a maximal subgroup $T$ such that $M=D T$ and $D \cap T \neq 1$.

In view of Claim (4), there is a maximal subgroup $T$ of $M$ such that $M=D T$. Assume that $D \cap T=1$. Then $D$ is a minimal normal subgroup of $M$. Note also that

$$
G / R \simeq M R / R \simeq M / M \cap R=M / D \simeq T
$$

is soluble by Claim (1). Then $D$ is a abelian group by Lemma 3.2, which contradicts Claim (4). Hence $D \cap T \neq 1$.

(6) At least one of the subgroups $T \cap R$ or $D=M \cap R$ is subnormal in $G$.

By hypothesis, a maximal chain $T<M<G$ contains a proper subgroup $L$ of $G$ such that some $G$-trace of $L$ is subnormal. In view of Claim (1), $R / 1$ is the unique $G$-boundary factor of $L$. Therefore, $L \cap R$ is subnormal in $G$ by [14, Ch.A, 14.2] Final contradiction.

By [14, A, 14.3], $R$ normalizes every subnormal subgroup of $G$. Hence, in view of Claim (6), either $R \leq N_{G}(T \cap R)$ or $R \leq N_{G}(D)$. Then since $(T \cap R)^{G}=$ $(T \cap R)^{R M} \leq(T \cap R)^{M} \leq D^{M} \leq M_{G}$ and $1 \neq D \cap T \leq T \cap R$, we obtain 
that $M_{G} \neq 1$. But this contradicts Claim (2). The final contradiction completes the proof.

Question 3.3 Is it true that $G$ is p-soluble if and only if every maximal chain of $G$ of length 2 contains a proper subgroup $M$ of $G$ such that either some $G$-trace of $M$ is subnormal or every $G$-boundary factor of $M$ is a $p^{\prime}$-group?

2. It is clear that every trace of any maximal subgroup of a soluble group is abelian. This fact is a motivation for our next observations, which can be proved similarly as Theorem 3.1.

Theorem 3.4 $G$ is soluble if and only if every maximal subgroup of $G$ has a nilpotent trace.

Corollary 3.5 (O. Yu. Schmidt [14, Ch.A, 10.7]). If every maximal subgroup of $G$ is nilpotent, then $G$ is soluble.

Nevertheless, we do not know the answer to the following

Question 3.6 Suppose that every maximal subgroup of $G$ has a supersoluble trace. Does it true then that $G$ is soluble?

3. It is well known that a $p$-soluble group is $p$-supersoluble if and only if for every its maximal subgroup $M$, we have that $|G: M|$ is either $p$ or a $p^{\prime}$-number [18, VI,9.2,9.3]. Note that if $G$ is $p$-soluble and $p$ divides the order of some boundary factor $H / M_{G}$ of a maximal subgroup $M$ of $G$, then $H / M_{G}$ is abelian $p$-group and so $\left|H / M_{G}\right|=|G: M|$. This elementary observation is a motivation for the following generalization of the Theorems 9.2 and 9.3 in [18].

Theorem $3.7 G$ is p-supersoluble if and only if for every maximal subgroup $M$ of $G$ such that $p$ divides the order of some boundary factor $H / M_{G}$ of $M$, we have $\left|H / M_{G}\right|=|G: M|$.

Proof See the proof of Theorem $C^{*}$.

Acknowledgments Research of the first author is supported by a NNSF grant of China (Grant \# 11371335) and Wu Wen-Tsun Key Laboratory of Mathematics, USTC, Chinese Academy of Sciences. Research of the second author supported by Chinese Academy of Sciences Visiting Professorship for Senior International Scientists (Grant No. 2010T2J12).

\section{References}

1. Johnson, D.L.: A note on supersoluble groups. Canadian J. Math. 23, 562-564 (1971)

2. Weinstein, M. (ed.): Between Nilpotent and Solvable. Polygonal Publishing House, Passaic (1982)

3. Birkhoff, G.: Lattices Theory, 3rd edn. AMS Coll. Publ., Providence (1984)

4. Schmidt, R.: Subgroup Lattices of Groups. Walter de Gruyter, Berlin (1994)

5. Ballester-Bolinches, A., Esteban-Romero, R., Asaad, M.: Products of Finite Groups. Walter de Gruyter, Berlin-New York (2010)

6. Shemetkov, L.A., Skiba, A.N.: On the $Х \Phi$-hypercentre of finite groups. J. Algebra 322, 2106-2117 (2009) 
7. Asaad, M.: Finite groups with certain subgroups of Sylow subgroups complemented. J. Algebra 323(7), 1958-1965 (2010)

8. Skiba, A.N.: On two questions of L. A. Shemetkov concerning hypercyclically embedded subgroups of finite groups. J. Group Theory 13, 841-850 (2010)

9. Li, B.: On П-property and П-normality of subgroups of finite groups. J. Algebra 334, 321-337 (2011)

10. Skiba, A.N.: A characterrization of hypercyclically embedded subgroups of finite groups. J. Pure Appl. Algebra 215, 257-261 (2011)

11. Guo, W., Skiba, A.N.: On $\mathcal{F} \Phi^{*}$-hypercentral subgroups of finite groups. J. Algebra 372, 275-292 (2012)

12. Yi, X., Alexander, N.: Skiba, some new characterizations of PST-groups. J. Algebra 399, 39-54 (2014)

13. Chen, X., Guo, W., Skiba, A.N.: Some conditions under which a finite group belongs a Baer local formation. Comm. Algebra 42, 4188-4205 (2014)

14. Doerk, K., Hawkes, T.: Finite Soluble Groups. Walter de Gruyter, Berlin-New York (1992)

15. Skiba, A.N.: Finite groups with given systems of generalized permutable subgroups. Proc. F.Scorina Gomel State Univ. 36(3), 12-31 (2006)

16. Ballester-Bolinches, A., Ezquerro, L.M., Skiba, A.N.: Local embeddings of some families of subgroups of finite groups. Acta Math. Sin. 25, 869-882 (2009)

17. Guo, X., Wang, J., Shum, K.P.: On semi-cover-avoiding maximal subgroups and solvability of finite groups. Comm. Algebra 34, 3235-3244 (2006)

18. Huppert, B.: Endliche Gruppen I. Springer-Verlag, Berlin, Heidelberg, New York (1967)

19. Deskins, W.E.: On maximal subgroups. Proc. Symp. Pure Math., Am. Math. Soc 1, 100-104 (1959)

20. Knyagina, B.N., Monakhov, V.S.: On $\pi^{\prime}$-properties of finite groups having a Hall $\pi$-subgroup. Sib. Math. J. 522, 309-398 (2011)

21. Huppert, B., Blackburn, N.: Finite Groups III. Springer-Verlag, Berlin-New York (1982)

22. Gorenstein, D.: Finite Groups. Harper \& Row Publishers, New York, Evanston, London (1968)

23. Guo, X., Shum, K.P.: Cover-avoidance properties and the structure of finite groups. J. Pure Appl. Algebra 181, 297-308 (2003)

24. Kurzweil, H., Stellmacher, B.: The Theory of Finite Groups: An Introduction. Springer-Verlag, New York-Berlin-Heidelberg (2004) 